\title{
Der ÄDA aktualisiert die Immuntherapie-Empfehlungen
}

m Jahr 1995 waren die letzten Empfehlungen des Ärzteverbandes Deutscher Allergologen (ÄDA) zur spezifischen Immuntherapie (Hyposensibilisierung) mit Allergenextrakten im ALLERGO JOURNAL erschienen. Inzwischen sind wesentliche Erkenntnisse zum positiven Einfluss der Hyposensibilisierung auf die bronchiale Hyperreagibilität, zur möglichen Einsparung von Medikamenten und zum Zugewinn an Lebensqualität hinzugekommen. Auch kommen unter der spezifischen Immuntherapie weniger neue Allergien hinzu und die Gefahr eines Etagenwechsels von der Rhinokonjunktivitis zum Asthma wird verringert. Aus diesen Gründen und weil zahlreiche plazebokontrollierte Studien die Effektivität der Immuntherapie noch mehr untermauert haben, erfreut sich die Immuntherapie heute größerer allgemeiner Wertschätzung.

Es hat sich inzwischen der Begriff „spezifische Immuntherapie (SIT)“" anstelle von De- oder Hyposensibilisierung mehr und mehr durchgesetzt. Auch wird der Vorschlag der WHO, von einer "AllergieImpfung" zu sprechen, heute gerne aufgegriffen.

Für die Praxis hat sich gegenüber früher geändert, dass am Tage der subkutanen Allergeninjektion sowohl vor als auch nach der Injektion starke körperliche Belastung zu vermeiden ist, was aus den umfangreichen Erkenntnissen zur „Exercise induced anaphylaxis" resultiert.

Die Allergenextrakte sollten vor der Injektion angewärmt werden, was am einfachsten durch Umgreifen der Spritze mit der Hand erfolgt. Dadurch werden eventuell vorhandene Aggregate in den Extrakten aufgelöst und es wird das Auftreten einer urtikariellen Reaktion nach der Injektion vermieden. Eine Dosisreduktion bei Anbruch einer neuen Flasche ist nicht mehr erforderlich. Die Extrakte gelten heute als so gut standardisiert und bei Kühlschranktemperatur als so gut haltbar, dass die bisher übliche Dosisreduktion nicht mehr für erforderlich erachtet wird.

Erste diskrete Hinweise auf eine erhöhte Anaphylaxie-Gefahr bei der Immuntherapie unter ACE-Hemmern fanden keine weitere Bestätigung, so dass wir uns den WHO-Richtlinien anschließen und diese nicht als Kontraindikation für die Immuntherapie betrachten.

$\mathrm{Neu}$ ist gegenüber den früheren Versionen der ÄDA-Empfehlungen auch der Warnhinweis des staatlichen Paul-Ehrlich Instituts für Sera und Impfstoffe, dass die Immuntherapie nur von allergologisch weitergebildeten bzw. allergologisch erfahrenen Ärzten verschrieben und angewendet werden darf. Hierauf wird heute in den Beipackzetteln eines jeden Allergenextrakts in Deutschland ausdrücklich hingewiesen.

Die sublinguale Immuntherapie wird heute sehr unterschiedlich beurteilt. Sie ist sicherlich nicht wirkungslos, allerdings sind weiterhin einige Fragen offen: Kontrolle der Compliance? Sicherheit? Wirksamkeit im Vergleich zur subkutanen Therapie? Ausreichend hohe Allergendosis bei einigen Präparaten auf dem Markt? Kosten-Nutzen-Relation? Wir haben uns daher zum jetzigen Zeitpunkt nicht für eine Empfehlung zur allgemeinen Anwendung dieser Therapieform entschließen können.
Bei der Notfallbehandlung empfehlen wir nicht mehr die Anwendung von Adrenalin zur Inhalation, da aufgrund einer sehr variablen Resorption keine Dosierungsempfehlung gegeben werden kann. Die Notfallbehandlung wurde durch Tabellen noch detaillierter als früher dargestellt, insbesondere sind jetzt auch Kinderdosierungen angegeben.

Die ÄDA-Empfehlungen stellen keine verbindlichen Richtlinien dar, erst recht keine Vorschriften, sondern lediglich die heutige Meinung der Mehrheit eines Expertengremiums des ÄDA.

Wir danken der Deutschen Gesellschaft für Allergologie und klinische Immunologie, insbesondere Herrn Prof. Dr. G. Schultze-Werninghaus, für die kritische Durchsicht des Textes und zahlreiche wertvolle Ergänzungen.

Prof. Dr. J. Sennekamp, Bonn

Dr. W. Kersten, Moers

Prof. Dr. L. Klimek, Wiesbaden 